\title{
Tight-binding model for borophene and borophane
}

\author{
M. Nakhaee, ${ }^{1,2, *}$ S. A. Ketabi, ${ }^{2, \dagger}$ and F. M. Peeters ${ }^{1, \ddagger}$ \\ ${ }^{1}$ Department of Physics, University of Antwerp, Groenenborgerlaan 171, B-2020 Antwerp, Belgium \\ ${ }^{2}$ School of Physics, University of Damghan, P.O. Box 36716-41167, Damghan, Iran
}

(Received 29 January 2018; published 21 March 2018)

\begin{abstract}
Starting from the simplified linear combination of atomic orbitals method in combination with first-principles calculations, we construct a tight-binding (TB) model in the two-centre approximation for borophene and hydrogenated borophene (borophane). The Slater and Koster approach is applied to calculate the TB Hamiltonian of these systems. We obtain expressions for the Hamiltonian and overlap matrix elements between different orbitals for the different atoms and present the SK coefficients in a nonorthogonal basis set. An anisotropic Dirac cone is found in the band structure of borophane. We derive a Dirac low-energy Hamiltonian and compare the Fermi velocities with that of graphene.
\end{abstract}

DOI: 10.1103/PhysRevB.97.125424

\section{INTRODUCTION}

A wide range of two-dimensional (2D) materials ranging from graphene to topological insulators [1-6] share the extraordinary phenomenon that electrons behave as relativistic particles in their low-energy excitations. This emergent behavior of fermions in condensed-matter systems has been classified as "Dirac materials," which have attracted both experimental and theoretical research.

Recently, a fully metallic boron-based 2D nanostructure has been synthesized on a silver crystal by physical vapor deposition, named borophene [7-11]. There are many phases of bulk and 2D boron allotropes such as $\alpha, \beta$, and so on, which have been proposed theoretically [12-14]. Although, boron avoids participation in the formation of chemical bonds to make a stable honeycomb lattice, it is possible to make a stable planar structure by a mixture of honeycomb together with triangular units $[15,16]$. This structure contains two atoms per primitive unit cell, which is called 2B:Pmmn, in which Pmmn stands for the space group 59 included in an orthorhombic crystal system. Xu et al. [17] predicted a novel Dirac material: hydrogenated borophene (borophane), exhibiting Dirac characteristics with a remarkable Fermi velocity which is nearly twice that of graphene.

Free-standing borophene has imaginary frequencies in its phononic dispersion and consequently is dynamically unstable, and therefore it needs a substrate to be stabilized. In contrast, borophane has a dynamically stable structure and densityfunctional theory (DFT) calculations showed that borophane has an anisotropic Dirac cone between the $\Gamma$ and $X$ points [17]. Considering the recent successful realizations of graphene, silicene, germanene, and stanene with similar structures, the predicted borophane with Dirac characteristics and ultrahigh Fermi velocity is an interesting $2 \mathrm{D}$ material. In contrast to

\footnotetext{
*mohammad.nakhaee@uantwerpen.be

†'saketabi@du.ac.ir

${ }^{\ddagger}$ francois.peeters@uantwerpen.be
}

group $X I V$ elements $(\mathrm{C}, \mathrm{Si}, \mathrm{Ge}, \mathrm{Sn})$ of the periodic table, borophene and borophane have no similar hexagonal honeycomb monolayer structures because of their electron deficiency [14].

DFT calculations have revealed that the Dirac cone in borophane, unlike for the group XIV elements [18], is not made up of only the $p_{z}$ orbital. In view of the position of the Dirac cone in the Brillouin zone and its lattice structure, one has to also take both $p_{x}$ and $p_{y}$ orbitals into account in the calculations.

The goal of the present paper is to evaluate the best Slater-Koster (SK) coefficients to generate the Dirac point in borophane within the tight-binding (TB) approximation with fitting to DFT results, and to obtain accurate results for the position of the Dirac cone and the velocity of the electrons at the Fermi level. The fitting approach is described in Sec. II, followed by an analysis of the energy spectrum around the Dirac cone in Sec. III.

\section{TIGHT-BINDING MODEL}

With the linear combination of atomic orbitals (LCAO) method, the system can be described by a set of noninteracting single particles. The SK scheme [19] is a powerful method to reproduce the first-principles data. The Naval Research Laboratory (NRL) scheme [20] is an extension of the SK method and an alternative method useful for systems including a large number of atoms in the unit cell. The SK scheme has been applied to construct the TB Hamiltonian of different systems. We follow the usual Levenberg-Marquardt nonlinear fitting algorithm [21] to find the best entries for both Hamiltonian and overlap matrices. The idea is to find the SK coefficients for the boron-boron bonds in borophene and use them as an initial guess for borophane, and then to find the parameters of the hydrogen-boron bonds to reproduce the Dirac cone.

For the borophene lattice with a basis of two boron atoms, one can assume a basis of four cubic harmonic orbitals [22] (one atomiclike $s$ and three $p_{x}, p_{y}$, and $p_{z}$ orbitals per atom), which generates a band structure with four valence and four 
conduction bands. In the case of borophane, two hydrogens will be added in the basis for which one can add one atomiclike $s$ orbital for each hydrogen. So we have ten bands, but we will focus on the first five bands. The starting point to construct the model is the following expression [23]:

$$
\sum_{v^{\prime}} \sum_{i^{\prime}}\left[H_{i v, i^{\prime} v^{\prime}}-\epsilon_{k} S_{i v, i^{\prime} v^{\prime}}\right] c_{i^{\prime} v^{\prime}}(k)=0,
$$

where

$$
\begin{gathered}
H_{i v, i^{\prime} v^{\prime}}=\left\langle\phi_{\nu}\left(r-r_{i}\right)|H| \phi_{\nu^{\prime}}\left(r-r_{i^{\prime}}\right)\right\rangle \\
S_{i v, i^{\prime} v^{\prime}}=\left\langle\phi_{v}\left(r-r_{i}\right) \mid \phi_{v^{\prime}}\left(r-r_{i^{\prime}}\right)\right\rangle .
\end{gathered}
$$

Here $H$ is the monoelectronic Hamiltonian and $S$ is the dimensionless overlap matrix with the basis formed by functions that are not orthonormal [24-26]. The integrals are calculated over the whole unit cell and $i$ and $v$ run over the atoms in the unit cell and the orbitals $s, p_{x}, p_{y}$, and $p_{z}$, respectively. Theoretically, the interatomic matrix elements mentioned in Eq. (3) can, in principle, be calculated directly from the known wave functions. In 1954, J. C. Slater and G. F. Koster [27] represented the expectation values of the Hamiltonian in the basis of the directed orbitals in terms of eight integrals $\left(v_{s s \sigma}\right.$, $\left.v_{s p \sigma}, v_{p p \sigma}, v_{p p \pi}, s_{s s \sigma}, s_{s p \sigma}, s_{p p \sigma}, s_{p p \pi}\right)$ as follows:

$$
\begin{aligned}
\langle s|H| s\rangle & =v_{s s \sigma}, \\
\left\langle s|H| p_{i}\right\rangle & =n_{i} v_{s p \sigma}, \\
\left\langle p_{i}|H| p_{j}\right\rangle & =\left(\delta_{i j}-n_{i} n_{j}\right) v_{p p \pi}+n_{i} n_{j} v_{p p \sigma},
\end{aligned}
$$

where $n_{i}=\mathbf{r} . \mathbf{e}_{i} /|\mathbf{r}|$ is the directional cosine in which $\mathbf{r}$ is the vector along the bond and $i$ runs over $x, y$, and $z$. The corresponding expressions for the overlap matrix can be found by replacing $H$ by $S$ and $v$ by $s$. To evaluate complex conjugated hopping matrix elements, we use the rule of angular quantum number: $\left\langle l|H| l^{\prime}\right\rangle=(-1)^{l+l^{\prime}}\left\langle l^{\prime}|H| l\right\rangle$.

In practice, the unknown parameters are determined by a best fitting of the energy bands that are obtained by other methods. We calculate the values of the eight integrals up to distances of the third nearest-neighbor sites.

In this paper, the electronic properties of borophene and borophane are investigated by first-principles calculations, which are used to fit the TB parameters. Atomic structure relaxations and calculations of electronic properties were performed using the OpenMX package [28] within the linear combination of the pseudoatomic orbitals method [29]. Structure relaxations were carried out using the quasi-Newton scheme till the forces on the atoms become less than $10^{-5} \mathrm{eV} / \mathrm{A}$. The exchangecorrelation energy was treated within the generalized gradient approximation, using the Perdew, Burke, and Ernzerhof functional [30,31]. A kinetic cutoff energy of $400 \mathrm{eV}$ for the plane-wave basis was adopted. Brillioun zone integrations were evaluated with the Monkhorst-Pack mesh $(22 \times 15 \times 1)$.

The fully relaxed borophene and borophane layers are displayed in Fig. 1. The optimized atomic positions and lattice constant for both borophene ( $a=1.62 \AA$ and $b=2.85 \AA$ ) and borophane ( $a=1.92 \AA$ and $b=2.81 \AA)$ are in agreement with previous works $[14,17]$. It should be noted that the lattice constant $a$ of borophane is remarkably increased with about $18.4 \%$ as compared with that of borophene, while the lattice (a)

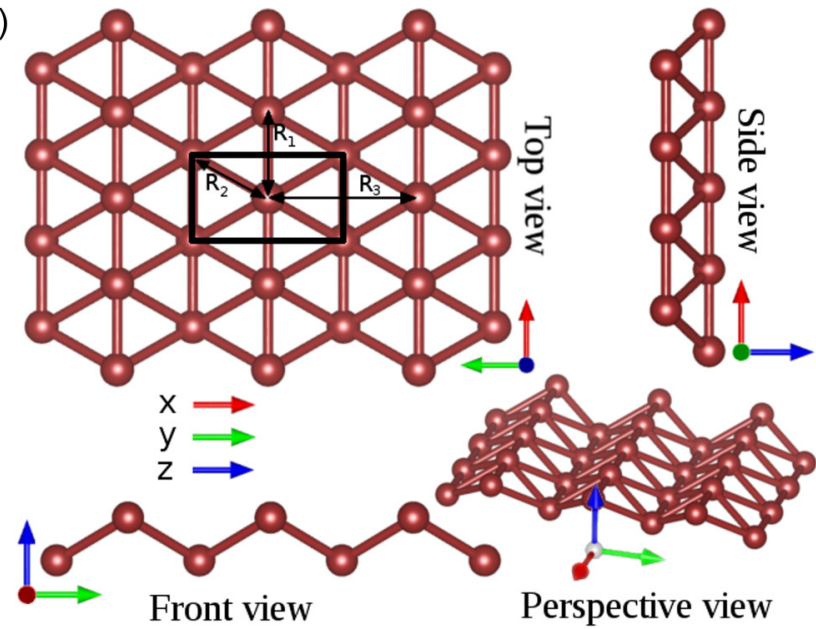

(b)

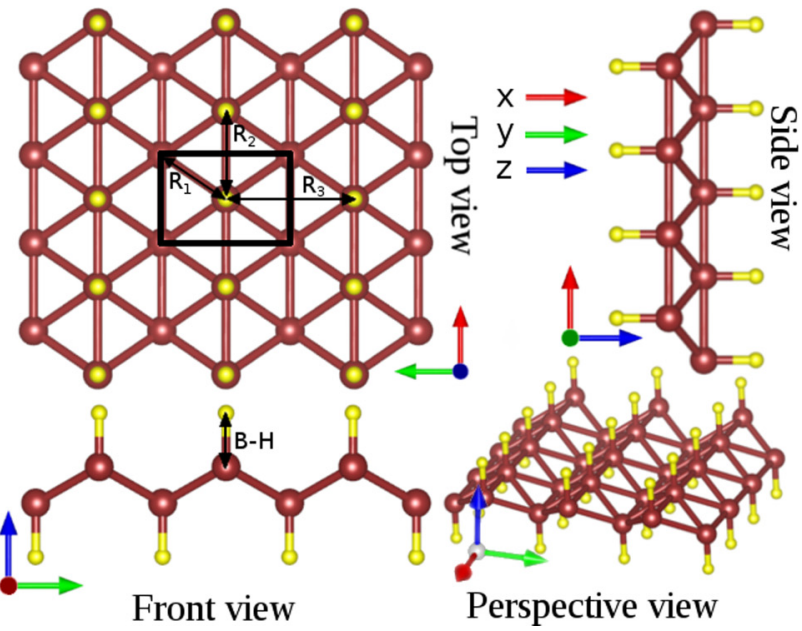

FIG. 1. The optimized structure of (a) borophene and (b) borophane. Black rectangle refers to the unit cell. Red (yellow) dots are boron (hydrogen) atoms. $R_{i}$ denotes the distance between boron atoms and H-B means the length of the hydrogen-boron bond.

vector $b$ is practically unchanged and as a result, the $B-B$ bond length in borophene is stretched, which consequently will affect the electrical properties along the $x$ direction.

To calculate the TB Hamiltonian by using the eight SK integrals, one needs to know the distance between the atoms. As shown in Fig. 1, the distance between two boron atoms is indicated by $R_{i}$, in which $i$ runs over three types of bonds between boron atoms and H-B is the length of the hydrogenboron bond. After optimizing the atomic positions one finds the distances $R_{1}=1.625 \AA, R_{2}=1.851 \AA$, and $R_{3}=2.852 \AA$ for borophene and $R_{1}=1.881 \AA, R_{2}=1.923 \AA, R_{3}=2.806 \AA$ and $\mathrm{H}-\mathrm{B}=1.189 \AA$ for borophane. Note that $R_{1}$ and $R_{2}$ are interchanged when going from borophene to borophane [see Figs. 1(a) and 1(b)].

The band structure of both systems is displayed in Fig. 2 . Note that the bands of both systems are formed by cubic harmonic orbitals $s, p_{x}, p_{y}$, and $p_{z}$ and, as shown, the Dirac cone for borophane (right figure) is formed by the intersection between $p_{x}$ and $p_{y}$ bands.

In contrast to group $X I V$ sheets, which have a Dirac cone formed by the $p_{z}$ orbital, in borophene and borophane the 

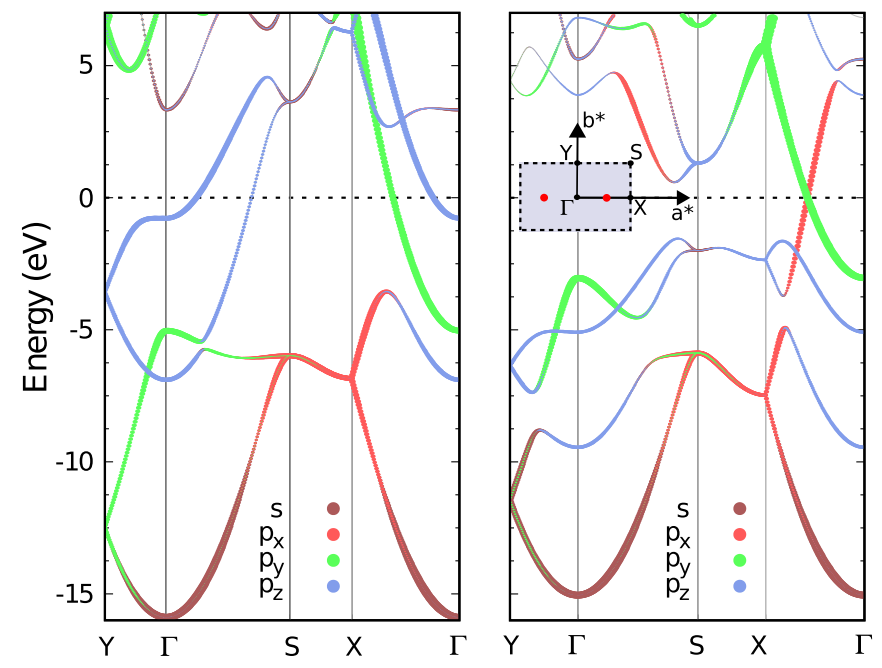

FIG. 2. The band structure of borophene (left side) and borophane (right side). Red points in the Brillouin zone (see inset in right figure) indicate the positions of the Dirac cones and $a^{*}=\frac{2 \pi}{a} \hat{a}$ and $b^{*}=\frac{2 \pi}{b} \hat{b}$ are the reciprocal lattice vectors.

contributions of other orbitals in the valence and conduction bands are significant. Considering that we want to construct a TB model up to three neighbors, a good TB fitting can only be achieved if the orbitals on the different sites are assumed to be nonorthogonal, as is common practice. The complicated electronic dispersion bands and the compact structure of borophene exhibit a $s p^{3}$ hybridization. Note that the first nearest neighbor of a boron atom in borophene lies at the nearest cell along $a$ and not in the primitive cell [see Figs. 1(a) and 1(b)]. For both structures, we have found the SK integrals of boron-boron bonds up to the third nearest neighbors, which results in a satisfactory fitting of the band structure (see Fig. 3). In hydrogenated borophene, we add an $s$ orbital in the position of the hydrogen atom and find its on-site and SK parameters related to the first-nearest boron atom in the lattice. There are nine parameters, which define the hoppings of an electron between the boron atoms and hydrogen located on top or below
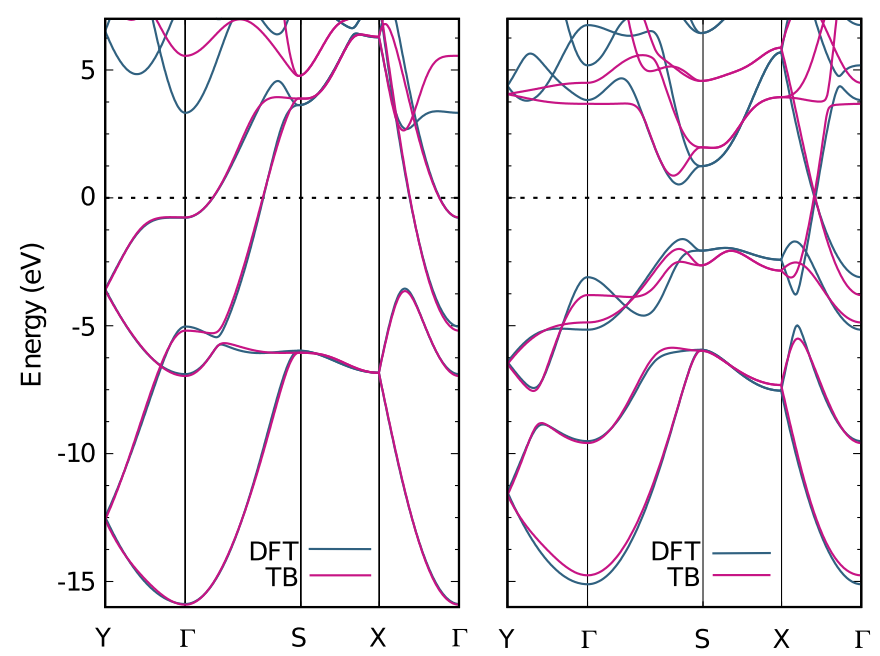

FIG. 3. The fitted tight-binding band structure of borophene (left side) and borophane (right side).
TABLE I. The Slater-Koster parameters for borophene (top) and borophane (bottom). The $V$ parameters are in $e V$, and the $S$ parameters are dimensionless.

\begin{tabular}{lcccccccc}
\hline \hline$R$ & $V_{s s \sigma}$ & $V_{s p \sigma}$ & $V_{p p \sigma}$ & $V_{p p \pi}$ & \multicolumn{1}{c}{$S_{s s \sigma}$} & \multicolumn{1}{c}{$S_{s p \sigma}$} & $S_{p p \sigma}$ & \multicolumn{1}{c}{$S_{p p \pi}$} \\
\hline $\mathrm{R}_{1}$ & -3.728 & -4.391 & 4.445 & -2.298 & 0.213 & 0.287 & -0.384 & 0.063 \\
$\mathrm{R}_{2}$ & -2.410 & -3.701 & 3.575 & -0.815 & 0.019 & 0.168 & -0.235 & -0.037 \\
$\mathrm{R}_{3}$ & -0.279 & -0.825 & 1.914 & -0.543 & -0.034 & -0.022 & 0.017 & 0.034 \\
$R$ & $V_{s s \sigma}$ & $V_{s p \sigma}$ & $V_{p p \sigma}$ & $V_{p p \pi}$ & $S_{s s \sigma}$ & $S_{s p \sigma}$ & $S_{p p \sigma}$ & \multicolumn{1}{l}{$S_{p p \pi}$} \\
\hline $\mathrm{R}_{1}$ & -2.822 & 2.987 & 2.290 & -0.672 & -0.0490 & -0.007 & -0.147 & 0.010 \\
$\mathrm{R}_{2}$ & -0.742 & 1.709 & 2.076 & -1.381 & 0.150 & -0.212 & -0.421 & 0.049 \\
$\mathrm{R}_{3}$ & -0.064 & 0.692 & 0.993 & -0.428 & 0.062 & -0.102 & -0.056 & 0.037 \\
$\mathrm{H}-\mathrm{B}$ & 4.523 & -3.941 & 0.000 & 0.000 & -0.113 & -0.347 & 0.000 & 0.000 \\
\hline \hline
\end{tabular}

the boron layer. From a view of the location of the hydrogen atoms, one can figure out that the $x$ and $y$ directional cosines are zero and, accordingly, we have to determine an addition of five nonzero parameters for borophene.

In Table I, we list the SK parameters of both systems in terms of bond length and bond type as obtained by fitting the DFT energy bands shown in Fig. 3. The rather large overlap values are an indication for the short interatomic distance between the boron atoms. The on-site parameters of boron and hydrogen atoms are presented in Table II. Using the SK parameters, one can calculate the Hamiltonian and the overlap matrix in terms of hoppings between different orbitals (see Supplemental Material [32]).

\section{LOW-ENERGY TWO-BAND EFFECTIVE HAMILTONIAN FOR BOROPHANE}

Concerning the Dirac cone found in the borophane band structure between the $\Gamma$ and $X$ points, one more simplification will be made. We will construct the analog of the Dirac equation for relativistic spin one-half particles within the two-band effective massless Dirac Hamiltonian that is valid for low energies. The corresponding Hamiltonian for the Dirac cone located at $\mathbf{k}_{d}=( \pm 0.64,0,0) \AA^{-1}$ is given by the following equation:

$$
H_{D}=v_{x} \sigma_{x} p_{x}+v_{y} \sigma_{y} p_{y}+v_{t} I p_{x}
$$

where $\sigma_{x}$ and $\sigma_{y}$ are the Pauli matrices and $I$ is the identity matrix of size 2. This expression defines a general anisotropic 2D Dirac cone, which is described by three constants $v_{x}, v_{y}, v_{t}$, which stand for the velocity in the $x$ and $y$ directions and the degree of tilting in the $x$ direction, respectively. Diagonalizing

TABLE II. The on-site energies for borophene and borophane in units of $\mathrm{eV}$.

\begin{tabular}{|c|c|c|c|c|c|c|c|c|}
\hline & \multicolumn{4}{|c|}{ Borophene } & \multicolumn{4}{|c|}{ Borophane } \\
\hline & $s$ & $p_{x}$ & $p_{y}$ & $p_{z}$ & $s$ & $p_{x}$ & $p_{y}$ & $p_{z}$ \\
\hline $\begin{array}{l}\text { B } \\
\text { H }\end{array}$ & -4.949 & 1.601 & -1.298 & 2.997 & $\begin{array}{c}-3.131 \\
7.575\end{array}$ & 3.861 & 0.103 & -1.015 \\
\hline
\end{tabular}




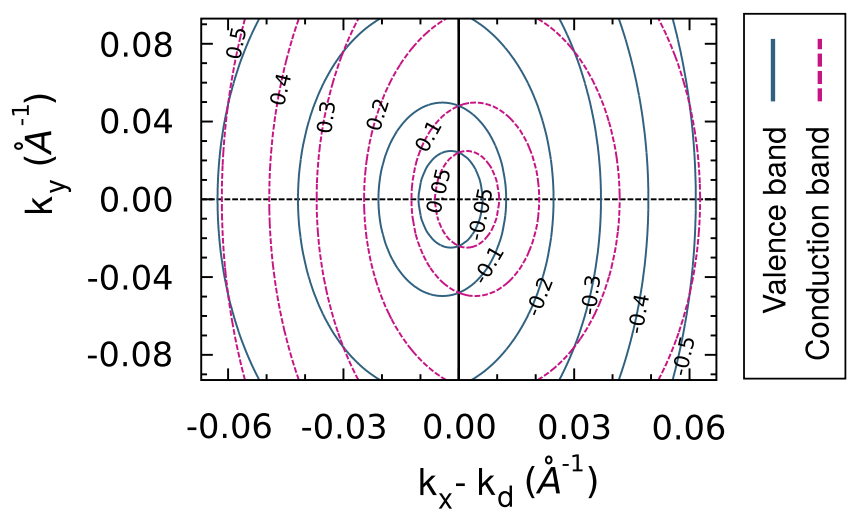

FIG. 4. Contour plot of conduction (red color) and valence (blue color) bands around the Dirac cone for borophane. Values of the contours are in $\mathrm{eV}$.

this Hamiltonian results in the energy dispersion

$$
\epsilon\left(k_{x}, k_{y}\right)=\left(k_{x}-k_{d}\right) v_{t} \pm \sqrt{\left(k_{x}-k_{d}\right)^{2} v_{x}^{2}+k_{y}^{2} v_{y}^{2}},
$$

where $v_{x}=19.58 \times 10^{5} \mathrm{~m} / \mathrm{s}, \quad v_{y}=6.32 \times 10^{5} \mathrm{~m} / \mathrm{s}, \quad v_{t}=$ $-5.06 \times 10^{5} \mathrm{~m} / \mathrm{s}$. A contour plot of the anisotropic Dirac cone is depicted in Fig. 4. The velocities in the positive and negative $x$ directions are given, respectively, by $v_{x}+\left|v_{t}\right|=$ $24.64 \times 10^{5} \mathrm{~m} / \mathrm{s}$ and $v_{x}-\left|v_{t}\right|=14.52 \times 10^{5} \mathrm{~m} / \mathrm{s} . v_{x}+\left|v_{t}\right|$, $v_{x}-\left|v_{t}\right|$, and $v_{y}$ are 2.95, 1.74, and 0.76 times the Fermi velocity of graphene $\left(v_{f}=8.36 \times 10^{5} \mathrm{~m} / \mathrm{s}\right)$.

The density of states per unit cell, derived from Eq. (4), is represented in Fig. 5, which is compared with the results obtained for graphene [33]. Despite the different shape of the Brillouin zone of borophane and graphene, they have approximately the same area and also the same number of Dirac cones per unit cell. The average of the Fermi velocities for low-energy fermions for borophane are higher than that of graphene. That is why the number of possible occupied states is less than that of graphene per unit cell. It is possible to derive an analytical expression for the Green's function and extract the imaginary part of it to calculate the density of states (see Appendix for more details). The density of states per unit cell corresponding to the energy dispersion Eq. (5) can

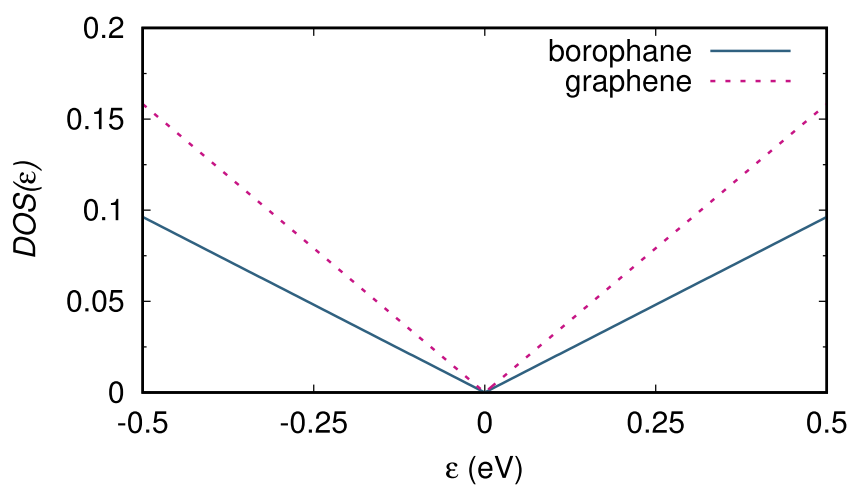

FIG. 5. Density of states per unit cell as a function of energy calculated from the low-energy Dirac Hamiltonian for borophane and graphene. be calculated as follows:

$$
\operatorname{DOS}(\varepsilon)=\frac{4 \pi v_{x}^{2}}{\Omega v_{y}\left(v_{x}^{2}-v_{t}^{2}\right)^{\frac{3}{2}}}|\varepsilon| \Theta\left(\varepsilon_{c}-|\varepsilon|\right),
$$

where $\Omega$ is the area of the Brillouin zone. The parameter $\varepsilon_{c}$ is a cutoff for the energy to make sure that Eq. (6) is valid within the linear regime of the band structure near the Dirac cones.

\section{SUMMARY}

To construct a TB model based on the simplified LCAO, we followed the SK approach in which the energy dispersion was fitted to the one obtained from DFT. We determined the SK coefficients in a nonorthogonal basis set for borophene and borophane, which allows one to calculate different electronic properties using Green's function theory, e.g., electrical transport, tunneling, effect of impurities, and defects on different electronic properties. We also calculated the Dirac lowenergy Hamiltonian of borophane, which describes the physics within the anisotropic Dirac cone, and derived an analytical expression for the density of states. The full expression for the Hamiltonian and overlap matrices for borophene and borophane are given in the supplementary information.

\section{ACKNOWLEDGMENTS}

Discussions with Dr. Vahid Derakhshan and M. A. M. Keshtan are gratefully acknowledged. This paper is supported by the Methusalem program of the Flemish government and the FLAT-ERA Project TRANS-2D-TMD.

\section{APPENDIX: ANALYTICAL EXPRESSION FOR THE GREEN'S FUNCTION FOR AN ANISOTROPIC DIRAC HAMILTONIAN}

Considering the Dirac equation mentioned in Sec. III, we calculate the Green's function for a general anisotropic Dirac cone which is tilted in the $x$ direction. We are interested in finding an analytical expression for the Green's function, which is needed to calculate the density of states. We start from the definition of the Green's function in the reciprocal representation as follows:

$$
G(\mathbf{k}, \varepsilon)=\left(\varepsilon I-H_{\mathbf{k}}\right)^{-1} .
$$

The density of states is defined as the imaginary part of the diagonal elements of the retarded Green's function. One can evaluate these components by using Eq. (4) and Eq. (A1) as below:

$$
G_{1,1}(\mathbf{k}, \varepsilon)=G_{2,2}(\mathbf{k}, \varepsilon)=\frac{\varepsilon-k_{x} v_{t}}{\left(\varepsilon-k_{x} v_{t}\right)^{2}-k_{x}^{2} \nu_{x}^{2}-k_{y}^{2} \nu_{y}^{2}} .
$$

It is useful to evaluate the real space Green's function $G(\mathbf{r}-$ $\mathbf{r}^{\prime}, \varepsilon$ ) by taking the Fourier transform. One motivation for calculating the Fourier transform of Green's function comes from the fact that we can easily derive the density of states from the imaginary part of the diagonal elements of Green's function in real space as follows:

$$
\operatorname{DOS}(\varepsilon)=\frac{-1}{\pi} \operatorname{Im}\left(G_{1,1}\left(\mathbf{r}=\mathbf{r}^{\prime}, \varepsilon\right)+G_{2,2}\left(\mathbf{r}=\mathbf{r}^{\prime}, \varepsilon\right)\right) .
$$


Corresponding real space Green's function is defined by the 2D Fourier transformation

$$
G\left(\mathbf{r}-\mathbf{r}^{\prime}, \varepsilon\right)=\frac{1}{\Omega} \int \mathrm{d} \mathbf{k}^{2} e^{i \mathbf{k} .\left(\mathbf{r}-\mathbf{r}^{\prime}\right)} G(\mathbf{k}, \varepsilon)
$$

where, $\Omega$ denotes the area of the first Brillouin zone. We define $k^{2}=\left(v_{x} k_{x}\right)^{2}+\left(v_{y} k_{y}\right)^{2}$ and $v_{x} k_{x}=k \cos \varphi$ and transform cartesian to polar coordinates.

$$
G_{1,1}(0, \varepsilon)=\frac{1}{\Omega} \int_{0}^{2 \pi} d \varphi \int_{0}^{k_{c}} k d k \frac{\varepsilon-\frac{\nu_{t}}{\nu_{x}} k \cos \varphi}{\left(\varepsilon-\frac{\nu_{t}}{v_{x}} k \cos \varphi\right)^{2}-k^{2}} .
$$

Here, $k_{c}=\varepsilon_{c} /\left(1-v_{t} / v_{x}\right)$, where $\varepsilon_{c}$ is the cutoff energy for evaluating the integral. After some simplifications, one can derive the Green's function expression as follows:

$$
G_{1,1}(0, \varepsilon)=\frac{1}{\Omega}(g(\varepsilon)+g(-\varepsilon)),
$$

with

$$
g(\varepsilon)=\frac{i \pi v_{x}}{v_{y}\left(v_{t}^{2}-v_{x}^{2}\right)}\left(\sqrt{-\varepsilon^{2}}-\sqrt{\frac{k_{c}^{2} v_{t}^{2}}{v_{x}^{2}}-\left(k_{c}-\varepsilon\right)^{2}}+\frac{\varepsilon v_{x}}{\sqrt{v_{t}^{2}-v_{x}^{2}}} \log \frac{v_{x} \sqrt{v_{t}^{2}-v_{x}^{2}} \sqrt{\frac{k_{c}^{2} v_{t}^{2}}{v_{x}^{2}}-\left(k_{c}-\varepsilon\right)^{2}+k_{c}\left(v_{t}^{2}-v_{x}^{2}\right)+\varepsilon v_{x}^{2}}}{\sqrt{-\varepsilon^{2}} v_{x} \sqrt{v_{t}^{2}-v_{x}^{2}}+\varepsilon v_{x}^{2}}\right) .
$$

To extract the imaginary part of the Green's function, we applied the Cauchy principal value $\frac{1}{x+i \eta}=P\left(\frac{1}{x}\right)-i \pi \delta(x)$ in which $\eta$ is an infinitely small quantity. After using the property of the Dirac delta function for the integrals over $k$ and $\varphi$, the imaginary part of Eq. (A6) becomes

$$
\operatorname{Im}\left(G_{1,1}(0, \varepsilon)\right)=\frac{-\pi^{2} v_{x}^{2}}{\Omega v_{y}\left(v_{x}^{2}-v_{t}^{2}\right)^{\frac{3}{2}}}|\varepsilon| \Theta\left(\varepsilon_{c}-|\varepsilon|\right)
$$

[1] B. A. Bernevig and T. L. Hughes, Topological Insulators and Topological Superconductors (Princeton University Press, New Jersey, USA, 2013).

[2] F. D. M. Haldane, Phys. Rev. Lett. 61, 2015 (1988).

[3] H. Zhang, C.-X. Liu, X.-L. Qi, X. Dai, Z. Fang, and S.-C. Zhang, Nat. Phys. 5, 438 (2009).

[4] Y. Xia, D. Qian, D. Hsieh, L. Wray, A. Pal, H. Lin, A. Bansil, D. Grauer, Y. S. Hor, R. J. Cava, and M. Z. Hasan, Nat. Phys. 5, 398 (2009).

[5] C. L. Kane and E. J. Mele, Phys. Rev. Lett. 95, 146802 (2005).

[6] V. Derakhshan and S. A. Ketabi, Physica E 85, 253 (2017).

[7] A. J. Mannix, X.-F. Zhou, B. Kiraly, J. D. Wood, D. Alducin, B. D. Myers, X. Liu, B. L. Fisher, U. Santiago, J. R. Guest, M. J. Yacaman, A. Ponce, A. R. Oganov, M. C. Hersam, and N. P. Guisinger, Science 350, 1513 (2015).

[8] B. Feng, J. Zhang, Q. Zhong, W. Li, S. Li, H. Li, P. Cheng, S. Meng, L. Chen, and K. Wu, Nat. Chem. 8, 563 (2016).

[9] Z. H. Zhang, A. J. Mannix, Z. L. Hu, B. Kiraly, N. P. Guisinger, M. C. Hersam, and B. I. Yakobson, Nano Lett. 16, 6622 (2016).

[10] Q. Zhong, L. Kong, J. Gou, W. Li, S. Sheng, S. Yang, P. Cheng, H. Li, K. Wu, and L. Chen, Phys. Rev. Mater. 1, 021001 (2017).

[11] Q. Zhong, J. Zhang, P. Cheng, B. Feng, W. Li, S. Sheng, H. Li, S. Meng, L. Chen, and K. J. Wu, J. Phys.: Condens. Matter 29, 095002 (2017).

[12] A. Lopez-Bezanilla and P. B. Littlewood, Phys. Rev. B 93, 241405 (2016).

[13] N. G. Szwacki, Nanoscale Res. Lett. 3, 49 (2008).

[14] Z. A. Piazza, H. S. Hu, W. L. Li, Y. F. Zhao, J. Li, and L. S. Wang, Nat. Commun. 5, 3113 (2014).

[15] H. Tang and S. Ismail-Beigi, Phys. Rev. Lett. 99, 115501 (2007).
[16] H. Tang and S. Ismail-Beigi, Phys. Rev. B 80, 134113 (2009).

[17] L. C. Xu, A. Du, and L. Kou, Phys. Chem. Chem. Phys. 18, 27284 (2016).

[18] C. C. Liu, H. Jiang, and Y. Yao, Phys. Rev. B 84, 195430 (2011).

[19] J. C. Slater and G. F. Koster, Phys. Rev. 94, 1498 (1954).

[20] D. A. Papaconstantopoulos, Handbook of the Band Structure of Elemental Solids (Plenum Press, New York, 1986).

[21] K. Levenberg, Q. Appl. Math. 2, 164 (1944).

[22] T. Kwiatkowski, S. Olszewski, and A. Wierzbicki, Int. J. Quantum Chem. 11, 21 (1977).

[23] W. C. Lu, C. Z. Wang, K. Ruedenberg, and K. M. Ho, Phys. Rev. B 72, 205123 (2005).

[24] C. M. Goringe, D. R. Bowler, and E. Hernandez, Rep. Prog. Phys. 60, 1447 (1997).

[25] W. Kutzelnigg, J. Mol. Struct (THEOCHEM) 169, 403 (1988).

[26] S. D. Deshpande and R. B. Pode, Am. J. Phys. 56, 362 (1988).

[27] D. A. Papaconstantopoulos and M. J. Mehl, J. Phys.: Condens. Matter 15, R413, (2003).

[28] S. Boker, M. Neale, H. Maes, M. Wilde, M. Spiegel, T. Brick, J. Spies, R. Estabrook, S. Kenny, T. Bates, P. Mehta, and J. Fox, Psychometrika 76, 306 (2011).

[29] R. W. Jansen and O. F. Sankey, Phys. Rev. B 36, 6520 (1987).

[30] J. P. Perdew, K. Burke, and M. Ernzerhof, Phys. Rev. Lett. 77, 3865 (1996).

[31] A. D. Becke, Phys. Rev. A 38, 3098 (1988).

[32] See Supplemental Material at http://link.aps.org/supplemental/ 10.1103/PhysRevB.97.125424 for the Hamiltonian and the overlap matrix in terms of hoppings between different orbitals for borophene and borophane.

[33] A. C. Neto, F. Guinea, N. M. Peres, K. S. Novoselov, and A. K. Geim, Rev. Mod. Phys. 81, 109 (2009). 